\title{
HEMIPARESIS AND LATERALIZED CORTICAL LESIONS ASSOCIATED TO SEVERE HYPOGLYCEMIA
}

\author{
Marcus Sabry Azar Batista', Kelson James Silva Almeida', \\ Marcelo Cunha Avelino ${ }^{3}$, Luciana Marinho Viana Borges ${ }^{3}$
}

Severe hypoglycemia may occur as a complication of insulin therapy or as a consequence of accidental or suicidal insulin injections'. It may result in a variety of neurological symptoms such as focal deficits, epileptic crisis, coma state and death. There is a relative rarity of reports about hypoglycemic-induced brain lesions ${ }^{2}$. In newborns, the parieto-occipital region is the most affected ${ }^{3}$. In adults, there is a greater variability, with reports of lesions in basal ganglia, hippocampus, temporal and occipital gray matter, insula cortex, corona radiata and splenium of the corpus callosum ${ }^{1,4}$.

The aim of this study is to report the case of a patient presenting coma, with left hemiparesis and brain lesions detected by computerized tomography scan (CT) and conventional magnetic resonance imaging (MRI). He had insulin-dependent diabetes mellitus and had a record of severe hypoglycemic episode.

\section{CASE}

A 31-year-old male, insulin-dependent diabetic patient, which left his home after a family brawl, at about 8 o'clock p.m when he had a capillary glycemia, which resulted $212 \mathrm{mg} \%$. He was met unconscious in the morning by his wife at a car, near their home, with many empty flasks and syringes of insulin. The last register of glycaemia in the dosimeter was $34 \mathrm{mg} \%$, at about 3 o'clock a.m.

He was taken to the hospital by the urgency medical care service. On admission, he showed stable vital signs and capillary glycemia of $82 \mathrm{mg} \%$. On neurological examination, the patient was comatose and with left hemiparesis. He was submitted to orotracheal intubation, mechanical ventilation and supportive care. A cerebral computerized tomography (CT) scan showed a slight parietal high-density area and MRI (Figure) showed cortical lesions with hypointensity and hyperintensity in T1- and T2weighted images, respectively, affecting the insula bilaterally, but more evident on the right side, the hippocampus (more accentuated on the right side), the medial part of frontal lobe, bilaterally but more accentuated on the right side, and the parietal lobe, almost exclusively on the right side.
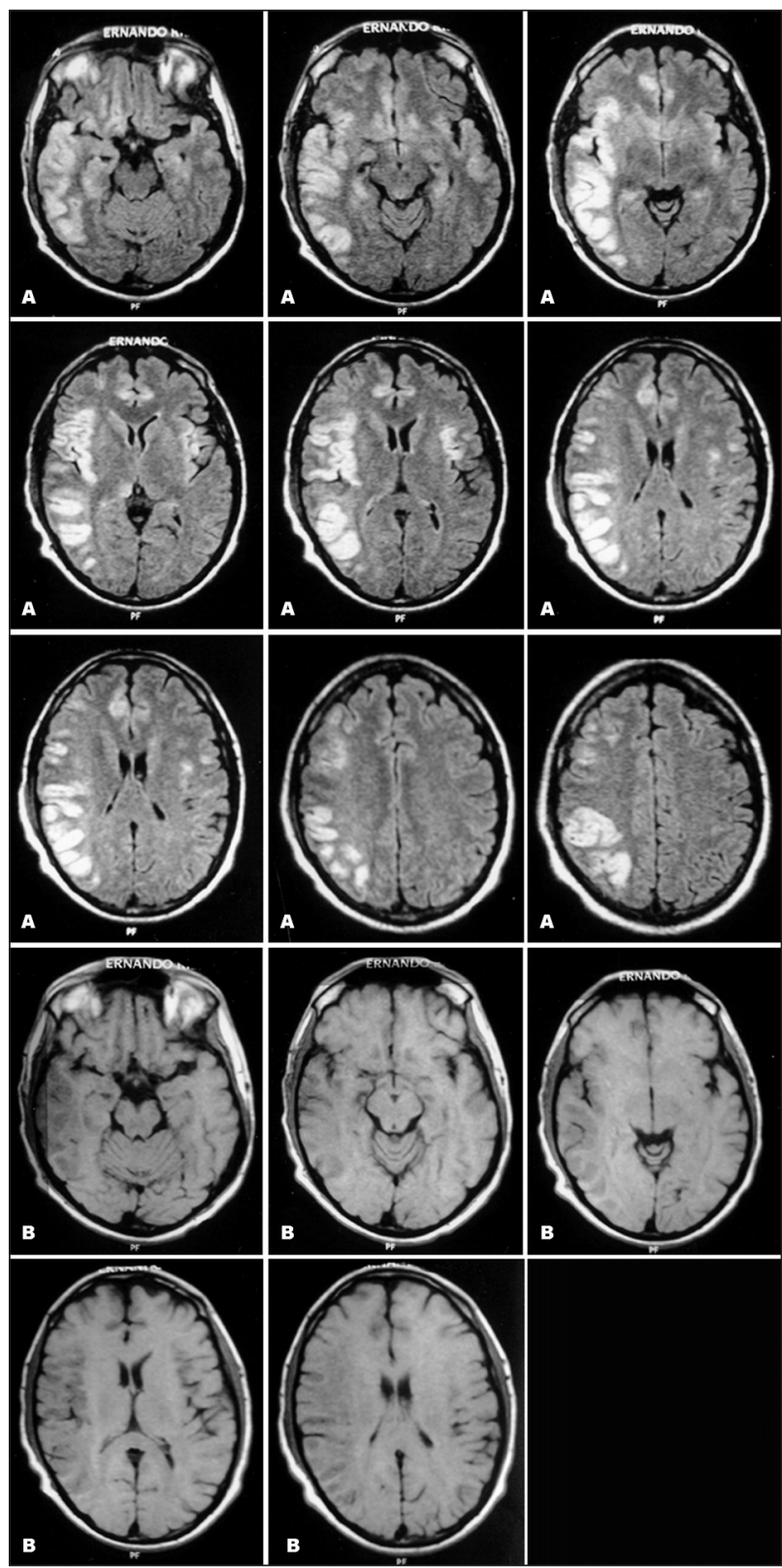

Figure. MRI showing cortical lesions with hyperintensity and hypointensity in T2- (A) and T7- (B) weighted images, respectively.

\section{HEMIPARESIA E LESÕES CORTICAIS LATERALIZADAS ASSOCIADAS À HIPOGLICEMIA GRAVE}

${ }^{1}$ Neurology Professor of the Federal University of Piauí, Piauí PI, Brazil (UFPI); ${ }^{2}$ Medical School Student, UFPI. Getúlio Vargas Hospital; ${ }^{3}$ Radiologist, Prontomed Hospital, Teresina PI, Brazil.

Received 27 July 2007, received in final form 8 October 2007. Accepted 28 November 2007.

Dr. Marcus Sabry Azar Batista - Rua Olavo Bilac 1737 -64001-020 Teresina PI - Brasil. E-mail: marcussabry@uol.com.br 
He evolved with progressive recovery of consciousness after a week, regaining temporospatial orientation gradually. The patient keeps memory for new facts and he has been slowly recovering the memory for events previous to the episode. He fully recovered from left hemiparesis. After three months of follow up, he did not return to his professional activity (teacher in a university) but he has been increasingly remembering a portion of the content of the subject he teaches. This study was approved by the Local Ethical Committee (Prontomed Hospital) after the informed consent by the patient.

\section{DISCUSSION}

Hypoglycemia in diabetic patients can occur because of dietetic errors, alcohol abuse, excessive physical effort or error in the dose of hypoglycemic drug, especially in elderly patients. Severe hypoglycemia, on the other hand, occurs when the insulin is used inadequately, because of an accident or suicidal or homicidal intentions ${ }^{1,2}$.

In newborns, hypoglycemia is the most common metabolic disorder, and there are many reports describing hypoglycemia-induced brain injury in this phase of life ${ }^{3}$. Neonatal hypoglycemia can result in long-term disabilities like epilepsy and visual impairment. The injuries include the parietal region frequently, but mainly the occipital region. The explanation for this selective vulnerability of occipital lobes in neonates is a regional deficit in expression and function of the glucose membrane transporter proteins $^{3}$.

In adults, differently from neonates, the severe hypoglycemia can result in injuries with a bigger variability of brain regions ${ }^{1}$, like in basal ganglia, hippocampus, insula, frontal regions and corpus callosum. Usually, patients are in coma or with respiratory failure; in the clinical outcome, entire disappearance of clinical features and even death have been reported due to hypoglycemic encephalopathy, deep memory loss and persistent vegetative state $^{1,4,5}$. Hemiparesis is a rare and/or under-diagnosed clinical manifestation of hypoglycemia ${ }^{6,7}$.

Dealing with a patient with acute onset of hemiparesis and coma, the possibility of stroke is the most probable $e^{4,8}$. In spite of this, patients with severe hypoglycemia usually have a normal glycemia at admission, which may delay the accurate recognition of etiology situation. Furthermore, diabetes mellitus is common among patients with vascular brain disease. In these circumstances, the clinical evolution and the MRI pictures can help in the differentiation between stroke and hemiparesis resultant of severe hypoglycemia.

In the hypoglycemic encephalopathy a reversion of brain lesions is more likely to take place than in stroke. Aoki et al. described reversible abnormalities in diffusionweighted MRI 10 days after glucose infusion ${ }^{9}$. In fact, as the reversible brain-imaging abnormalities and hemiparesis caused by hypoglycemia can mimic a transient ischemic attack or stroke ${ }^{10}$, the determination of blood glucose levels, the effects of its infusion and the comparison of MRI images on the day of admission to one taken several days after glucose infusion can be useful tools to make differential diagnosis?.

The mechanisms that explain the neuronal damage during hypoglycemic episodes involves cytotoxic oedema, shrinkage of the extracellular space and failure of ionic pumps of the cell membrane after energy depletion ${ }^{4,8}$. Additionally, it is developed a tissue alkalosis for ammonia formation from the deamination of amino acids and consumption of metabolic acids. The opposite occurs in regions of brain infarction where it is observed intracellular acidosis ${ }^{4,8}$.

Another considerable hypothesis is the disruption of protein synthesis in the superficial layers of the cortex, in the basal ganglia, and hippocampus ${ }^{1,9}$. On the other hand, the cerebellum and brain stem are less affected by this mechanism because they have effective strategies to transport glucose 9 . Hypoperfusion in injured areas of the cortex may occur for the selective regional loss of autoregulation in the cortex after hypoglycemia'. Another possibility is the occurrence of changes in blood-brain barrier induced by hypoglycemia resulting in brain oedema" ${ }^{11}$.

Mori et al. described the post-mortem examination in a patient with hypoglycemic encephalopathy that had evidence of high-intensity areas in brain white matter on diffusion weighted MRI. More than cortical laminar necrosis, neuronal necrosis in hippocampus and dentate gyrus (lesions compatibles with the brain injury induced by hypoglycemia), they observed that the cerebral white matter exhibited severe loss of myelin and that its axons had reactive astrocytosis and macrophage infiltration ${ }^{12}$.

Others neuroimaging techniques such as positron emission tomography (PET), functional magnetic resonance imaging ( $\mathrm{f}-\mathrm{MRI}$ ) and diffusion weighted MRI can provide maps of blood flow/oxygenation changes during the neuronal activity and the manner in which these are modulated by physiological factor, such as blood glucose level ${ }^{1,13,14}$

Anderson et al. induced a hypoglycemic state in ten healthy patients and studied, by the f-MRI that contrasted in dependence of blood oxygen level, the signs changes in visual cortex after passive visual stimulation. They observed that during moderate hypoglycemia, this method showed a reduced activity in the considered cortical area and they suggested also that in other regions of cortex, the variations in signal on $\mathrm{f}-\mathrm{MRI}$ could reveal the regional vulnerability of the brain to hypoglycemia ${ }^{13}$. 
It is reported that radiological findings caused by hypoglycemia can have time-related changes. Maekawa et al. evidenced lesions on diffusion weighted MRI at the cortex of the bilateral posterior lobes and hippocampus in a patient with severe hypoglycemia; these lesions were sustained for 7 days after the severe hypoglycemic episode. In the sequence, these findings lessened and disappeared at the fourteenth day after the insult. It is important to comment that at the day of images' reversion, the patient started opening his eyes in response to calling ${ }^{15}$.

The analysis of the brain images' changes after a severe hypoglycemic episode can be useful to predict the prognosis. Mainly the f-MRI can predict the patient's evolution for its sensitivity to detect the cytotoxic damage ${ }^{9}$. It has been described that the most patients with lesions on conventional MRI have a poor functional prognosis ${ }^{14}$. Basal ganglia involvement is also correlated with poor clinical outcome ${ }^{5}$. Nevertheless, it has been suggested that lesions in this region can be a result of the association of hypoglycemia with hypoxia'.

We supposed that the preservation of occipital cortex in our patient could have occurred for the absence of luminous stimulation in the place where the event happened; the impairment of parietal cortex and pre-motors areas can reflect the active regions during hypoglycemia.

This article contributes for the evaluation of severe hypoglycemia-induced changes in CT and MRI and for the understanding of selective susceptibility of cortical areas to severe hypoglycemia. For the early recognition and intervention, we consider essential to remember that severe hypoglycemia can be a possible cause of coma state and focal signs, to favor a differential diagnosis with a pos- sible stroke, especially in insulin-dependent patients or in those cases in which intentional insulin injection was suspected.

\section{REFERENCES}

1. Jung SL, Kim BS, Lee KS, Yoon KH, Byun JY. Magnetic resonance imaging and diffusion-weighted imaging changes after hypoglycemic coma. J Neuroimaging 2005;15:193-196.

2. Gasparetto EL, Bianchet LC, Davaus T, Carvalho A Neto. Intoxication with oral hypoglycemics as a cause of diffuse brain injury: case report. Arq Neuropsiquiatr 2006;64:1036-1038.

3. Filan PM, Inder TE, Cameron FJ, Kean MJ, Hunt RW. Neonatal hypoglycemia and occipital cerebral injury. J Pediatr 2006;148:552-555.

4. Fujioka M, Okuchi K, Hiramatsu KI, Sakaki T, Sakaguchi S, Ishii Y. Specific changes in human brain after hypoglycemic injury. Stroke 1997;28:584-587.

5. Finelli PF. Diffusion-weighted MR in hypoglycemic coma. Neurology 2001;57:933-935

6. Shirayama H, Ohshiro Y, Kinjo Y, et al. Acute brain injury in hypoglycaemia-induced hemiplegia. Diabet Med 2004;21:623-624.

7. Ozer H, Selcuk D, Albayram S. Acute brain injury with hemiparesis due to hypoglycaemia. Diabet Med 2005;22:1287-1288.

8. Hemelsoet DM. Acute brain injury in hypoglycaemia-induced hemiplegia Diabet Med 2005;22:1287.

9. Aoki T, Sato T, Hasegawa K, Ishizaki R, Saiki M. Reversible hyperintensity lesion on diffusion-weighted MRI in hypoglycemic coma. Neurology 2004;63:392-393.

10. Cordonnier C, Oppenheim C, Lamy C, Meder JF, Mas JL. Serial diffusion and perfusion-weighted MR in transient hypoglycemia. Neurology 2005;65:175

11. Berbel-Garcia A, Porta-Etessam J, Martinez-Salio A, et al. Transient cerebral oedema associated to hypoglycaemia. Rev Neurol 2004;39:10301033.

12. Mori F, Nishie M, Houzen H, Yamaguchi J, Wakabayashi K. Hypoglycemic encephalopathy with extensive lesions in the cerebral white matter. Neuropathology 2006;26:147-152.

13. Anderson AW, Heptulla RA, Driesen N, et al. Effects of hypoglycemia on human brain activation measured with fMRI. Magn Reson Imaging 2006;24:693-697.

14. Yanagawa Y, Isoi N, Tokumaru AM, Sakamoto T, Okada Y. Diffusionweighted MRI predicts prognosis in severe hypoglycemic encephalopathy. J Clin Neurosci 2006;13:696-699.

15. Maekawa S, Aibiki M, Kikuchi K, Kikuchi S, Umakoshi K. Time related changes in reversible MRI findings after prolonged hypoglycemia. Clin Neurol Neurosurg 2006;108:511-513. 